\title{
Effect of Platelet-Rich Plasma on Chondrogenic Differentiation in Three-Dimensional Culture
}

\author{
Steven Elder ${ }^{*}, 1$ and John Thomason ${ }^{2}$ \\ ${ }^{I}$ Department of Agricultural \& Biological Engineering, Bagley College of Engineering, Mississippi State University, \\ Starkville, MS, USA \\ ${ }^{2}$ Department of Clinical Sciences, College of Veterinary Medicine, Mississippi State University, Starkville, MS, USA
}

\begin{abstract}
Platelet-rich plasma (PRP) may have the potential to enhance articular cartilage regeneration through release of growth factors including transforming growth factor isoforms. The purpose of this study was to investigate the potential for PRP to stimulate chondrogenic differentiation in three-dimensional PRP hydrogel constructs. Allogenic PRP was prepared using a double centrifugation protocol which resulted in a platelet concentration approximately $250 \%$ above baseline. Canine marrow stromal cells were encapsulated at $6.8 \times 10^{6}$ cells $/ \mathrm{ml}$ in either $2 \%$ sodium alginate or in a $3: 1$ mixture of freshly prepared PRP and $2 \%$ alginate. PRP and alginate beads were cultured in chemically defined chondrogenic medium with and without $10 \mathrm{ng} / \mathrm{ml}$ TGF- $\beta 3$. PRP cultures were additionally supplemented with frozenthawed PRP. In the absence of TGF- $\beta 3$, PRP had a mild stimulatory effect on cell proliferation. PRP did not stimulate cell proliferation in the presence of TGF- $\beta 3$. Cells exposed to TGF- $\beta 3$ accumulated significantly more GAG/DNA than those which were not, but there was not a statistically significant difference between alginate and PRP. Total collagen content was greater in PRP than in alginate, regardless of TGF- $\beta 3$. Chondrogenesis in PRP was qualitatively and spatially different than that which occurred in conventional alginate beads and was characterized by isolated centers of intense chondrogenesis. Overall the results demonstrate that PRP alone weakly promotes chondroinduction of marrow stromal cells, and the effect is greatly augmented by TGF- $\beta 3$.
\end{abstract}

Keywords: Chondroinduction, hydrogel culture, mesenchymal stem cells, platelet-rich plasma.

\section{INTRODUCTION}

Enriching the platelet concentration in blood plasma, typically through centrifugation, yields platelet-rich plasma (PRP). Each platelet carries approximately 50-80 $\alpha-$ granules, the contents of which are released during platelet activation [1]. These contents include hundreds of soluble proteins, including platelet derived growth factor (PDGF), transforming growth factor-beta (TGF- $\beta$ ), and insulin-like growth factor (IGF) [2]. Such growth factors are known to stimulate proliferation, differentiation, and migration of mesenchymal stem cells. Thus PRP could be a source of concentrated autologous growth factors with the potential to stimulate musculoskeletal tissue regeneration.

PRP has been investigated as a means to accelerate healing and enhance regeneration of bone $[3,4]$, tendon [5, 6], ligament [7, 8], skeletal muscle [9], and cartilage [10-13]. With respect to cartilage, the results have been mixed. Wu et al. demonstrated that subcutaneous injection of auricular chondrocytes embedded in a PRP gel produced a tissue similar to that of native cartilage in a rabbit model [13], and Milano et al. found that a PRP fibrin glue gel improved the stiffness and histological grade of repair tissue in microfracture-treated chondral lesions in the sheep stifle

*Address correspondence to this author at the Department of Agricultural \& Biological Engineering, Mississippi State University, P.O. Box 9632, Mississippi State, MS 39762, USA; Tel: 662-325-9107;

E-mail: selder@abe.msstate.edu joint [10]. On the other hand, Kon et al. found that soaking a hydroxyapatite-collagen scaffold in autologous PRP interfered with its ability to support osteochondral regeneration [12].

Our long-term goal is to clarify the potential for PRP to improve approaches to cartilage tissue engineering based on chondroinduction of marrow-derived mesenchymal stem cells (MSCs). Several studies have shown that PRP stimulates proliferation of MSCs in monolayer [14-16], and the PRP-induced expansion does not diminish the MSCs' multilineage differentiation potential [17]. Some studies demonstrated that PRP can accelerate osteogenic differentiation in vitro, as evidenced by PRP-induced increases in alkaline phosphatase and mineralization [18-20], whereas others have observed no osteoinductive effect of PRP [21, 22]. There are far fewer published reports concerning the effect of PRP on chondrogenic differentiation, but the literature to date is somewhat encouraging. Mishra and colleagues demonstrated that non-activated PRP substantially upregulated gene expression of the chondrogenic markers Sox-9 and aggrecan in monolayer cultures of human MSCs [23]. Krüger et al. observed that exposure of human cortico-spongious progenitor cell pellets to frozen/thawed PRP increased proteoglycan and type II collagen staining and induced gene expression of aggrecan, collagen types II and IX, and link protein [24]. However, a study by Drengk and coworkers demonstrated an inhibitory effect of PRP on chondroinduction of ovine bone marrow MSCs in pellet culture [25]. The addition of PRP to culture 
medium containing $10 \%$ fetal bovine serum negatively influenced immunhistochemical staining for type II collagen.

The purpose of the current study was to investigate the potential for PRP to stimulate chondroinduction of MSCs in three-dimensional hydrogel culture. We hypothesized that cells embedded in PRP/alginate hydrogels and exposed to frozen-thawed PRP in the culture medium would exhibit superior chondroinduction to cells in alginate beads without PRP supplementation. A standard method of stimulating chondrogenic differentiation in vitro is by culturing MSCs in medium which contains TGF- $\beta 3$ inducer. The effects of PRP were therefore tested in the presence and absence of TGF- $\beta 3$ to facilitate comparison between the two and to explore the possibility that PRP could enhance TGF- $\beta 3$-mediated differentiation.

\section{METHODS}

All procedures involving live animals were conducted according to protocols approved by the Mississippi State Institutional Animal Care and Use Committee. Marrow cores were harvested from the proximal femurs of 3 mixed-breed dogs using a Jamshidi needle. Pooled cores were centrifuged to isolate the marrow, which was disrupted by pipetting and seeded onto tissue culture polystyrene in Dulbecco's Modified Eagle Medium (DMEM) containing 10\% fetal bovine serum (FBS). Twenty-four hours later non-adherent cells were removed by complete medium change. Adherent cells, hereafter referred to as mesenchymal stem cells (MSCs), were expanded to the third passage.

Blood collected from 2 mixed-breed dogs was pooled together. It was collected via jugular venipuncture with a 20 -gauge needle directly into $8.5 \mathrm{ml}$ vacutainer tubes containing acid citrate dextrose (ACD-A) anticoagulant. Blood was centrifuged at $1200 \times$ gravity (or $1200 \mathrm{~g}$ ) for 3 minutes to separate red blood cells from plasma. The upper plasma phase, including the interface, was centrifuged at $1000 \mathrm{~g}$ for 5 minutes to pellet the platelets. Two-thirds of the platelet-poor plasma supernatant was discarded, and the platelets were resuspended in the remaining third to create platelet-rich plasma.

Half the cells were used to establish alginate bead cultures. Sodium alginate was dissolved in DMEM at $2 \% \mathrm{wt} / \mathrm{vol}$ and sterilized by autoclaving. MSCs were suspended in alginate at a density of $6.8 \times 10^{6} \mathrm{cells} / \mathrm{ml}$, and this suspension was added dropwise to $100 \mathrm{mM} \mathrm{CaCl}_{2}$ in $0.9 \% \mathrm{NaCl}$. Following 5 minutes for polymerization, the beads were rinsed twice with phosphate-buffered saline and incubated with defined chondrogenic medium (DCM). Four wells of a 24-well plate each contained 6-8 beads and $2 \mathrm{ml}$ of medium (approximately 30 beads in total). The composition of DCM was as follows: high-glucose Dulbecco's modified Eagle's medium (DMEM) supplemented with $1 \%$ ITS+Premix (BD Biosciences), $0.1 \mathrm{mM}$ dexamethasone, $50 \mu \mathrm{g} / \mathrm{ml}$ ascorbate- 2 phosphate, $1 \mathrm{mM}$ sodium pyruvate, $40 \mu \mathrm{g} / \mathrm{ml} \mathrm{L}$-proline, and $1 \%$ antibiotic-antimycotic solution (Sigma-Aldrich, St. Louis, MO). Two of the wells containing half the beads $(\mathrm{n} \approx 15)$ were additionally supplemented with $10 \mathrm{ng} / \mathrm{ml}$ transforming growth factor-beta 3 or TGF- $\beta 3$ (Peprotech, Rocky Hill, NJ).
Half the cells were embedded in PRP beads. PRP was mixed with $2 \%$ alginate at a ratio of 3 parts PRP to 1 part alginate. MSCs were suspended in the mixture at a density of $6.8 \times 10^{6}$ cells $/ \mathrm{ml}$, and the mixture was added dropwise to a solution of $6 \% \mathrm{CaCl}_{2}$ and $0.9 \% \mathrm{NaCl}$. Approximately 30 beads were evenly distributed to 4 wells of a 24 -well plate containing $2 \mathrm{ml}$ of culture medium. PRP beads were treated in the same manner as alginate beads, with half in DCM without growth factor and half in DCM containing $10 \mathrm{ng} / \mathrm{ml}$ TGF- $\beta 3$ ( $\mathrm{n} \approx 15$ per group). PRP cultures were additionally supplemented with frozen-thawed PRP to 5\% of the total medium volume. Media were changed every third day, including frozen-thawed PRP. Supplementation with frozenthawed PRP was discontinued after 2 weeks.

Cell viability and chondrogenesis were assessed at 4 weeks. Cell viability was evaluated by fluorescence-based live/dead staining. A randomly selected bead from each group was stained using the PromokineLive/Dead Cell Staining Kit II (PromoCell GmbH, Heidelberg, Germany) according to the manufacturer's instructions. Images of stained cultures were captured on an Axiovert 200M Confocal Laser Scanning Microscope (Zeiss, Göttingen, Germany). Another 2 beads per group were fixed in $10 \%$ neutral buffered formalin and embedded in paraffin for sectioning. Sections were stained with $1 \%$ Toluidine blue or immunostained using SuperPicture ${ }^{\mathrm{TM}}$ 3rd Gen IHC Kit (Invitrogen Corporation, Camarillo, CA) to detect collagen types I and II. Sections to be immunostained were deparaffinized in xylene, rehydrated in graded ethanol, and incubated with $2 \mathrm{mg} / \mathrm{ml}$ hyaluronidase in PBS for 60 minutes at $37^{\circ} \mathrm{C}$. They were then incubated with $1 \mathrm{mg} / \mathrm{ml}$ pronase for 15 minutes at $37^{\circ} \mathrm{C}$ for antigen retrieval. A mouse monoclonal antibody to collagen type I (ab6308, Abcam, Cambridge, MA) or a mouse monoclonal antibody to collagen type II (II-II6B3, Developmental Studies Hybridoma Bank, University of Iowa) was applied for 24 hours at $4^{\circ} \mathrm{C}$. Both antibodies were diluted in PBS with $1 \%$ bovine serum albumin. The type I antibody was diluted 1:100 and the type II antibody (supernatant) was diluted $1: 10$. Following incubation in the primary antibody slides were stained according to the instructions of the kit.

Quantitative biochemistry was performed on 10 beads per treatment group. They were digested for 24 hours in papain at $60^{\circ} \mathrm{C}$ according to the method of Hoemann [26]. The digestate was assayed for DNA and glycosaminoglycan (GAG) by the Hoechst and DMB methods, respectively [26]. For the Hoechst procedure, fluorescence intensity was measured using a GloMax ${ }^{\circledR}$-Multi Jr Single Tube Multimode Reader (Promega Corporation, Madison, WI), and DNA content was determined from a standard curve created from calf thymus DNA. For the DMB procedure, optical absorbance at $530 \mathrm{~nm}$ and $590 \mathrm{~nm}$ was measured using a Micro Quant microplate spectrophotometer (BioTek Instruments, Winooski, VT), and GAG content was determined from a standard curve created with chondroitin4-sulfate. The remainder of each sample was then freeze dried and collagen content determined using the chloramine$\mathrm{T}$ assay [27]. Type I collagen from bovine skin was used as a standard. GAG and collagen contents were normalized to DNA. 
Quantitative data were statistically analyzed using a univariate ANOVA model in IBM SPSS Statistics 19, and p-values less than 0.05 were taken to indicate a significant difference.

\section{RESULTS}

Platelet counts showed that PRP contained 2.5 times the concentration of platelets in whole blood. Alginate beads maintained their original shape and size while becoming decreasingly translucent. PRP beads contracted and occasionally fused together. One sample in each experimental group was examined for cell viability by live/dead fluorescence assay. All samples contained a substantial proportion of dead cells. Live cells in PRP were found in clusters, and there was not a discernible difference between the bead which had been exposed to TGF- $\beta 3$ and the bead which had not. In alginate beads exposed to TGF- $\beta 3$ live dead cells were evenly dispersed among live cells, and the proportions were approximately equal. In the alginate bead not exposed to TGF- $\beta 3$, a single live cell was observed (Fig. 1). The individual beads randomly selected for viability staining may not have been representative of all beads subjected to the same experimental conditions.

Toluidine blue staining of the cells in alginate showed them to be generally spherical. They were occasionally located within lacunae and surrounded by a pericellular metachromatic extracellular matrix (ECM). Beads exposed to TGF- $\beta 3$ contained noticeably more multicellular clusters of cells surrounded by ECM (Fig. 2). In PRP without TGF- $\beta 3$ some of the cells displayed the morphology of chondrocytes and had accumulated metachromatic pericellular matrix, but most did not (Fig. 3A); and there were some extensive areas which were devoid of any overt chondrogenesis (Fig. 3B). The same was generally true in the presence of TGF- $\beta 3$. However, these beads also contained a few regions of the most robust chondrogenesis compared to all other groups, characterized by large cell clusters producing abundant ECM (Fig. 3C, D).

Across all groups, dense pericellular ECM displayed intense positive staining for collagen type II (Fig. 4). Some positive staining for type I collagen could also be detected in the ECM surrounding cells in the PRP beads (Fig. 5).

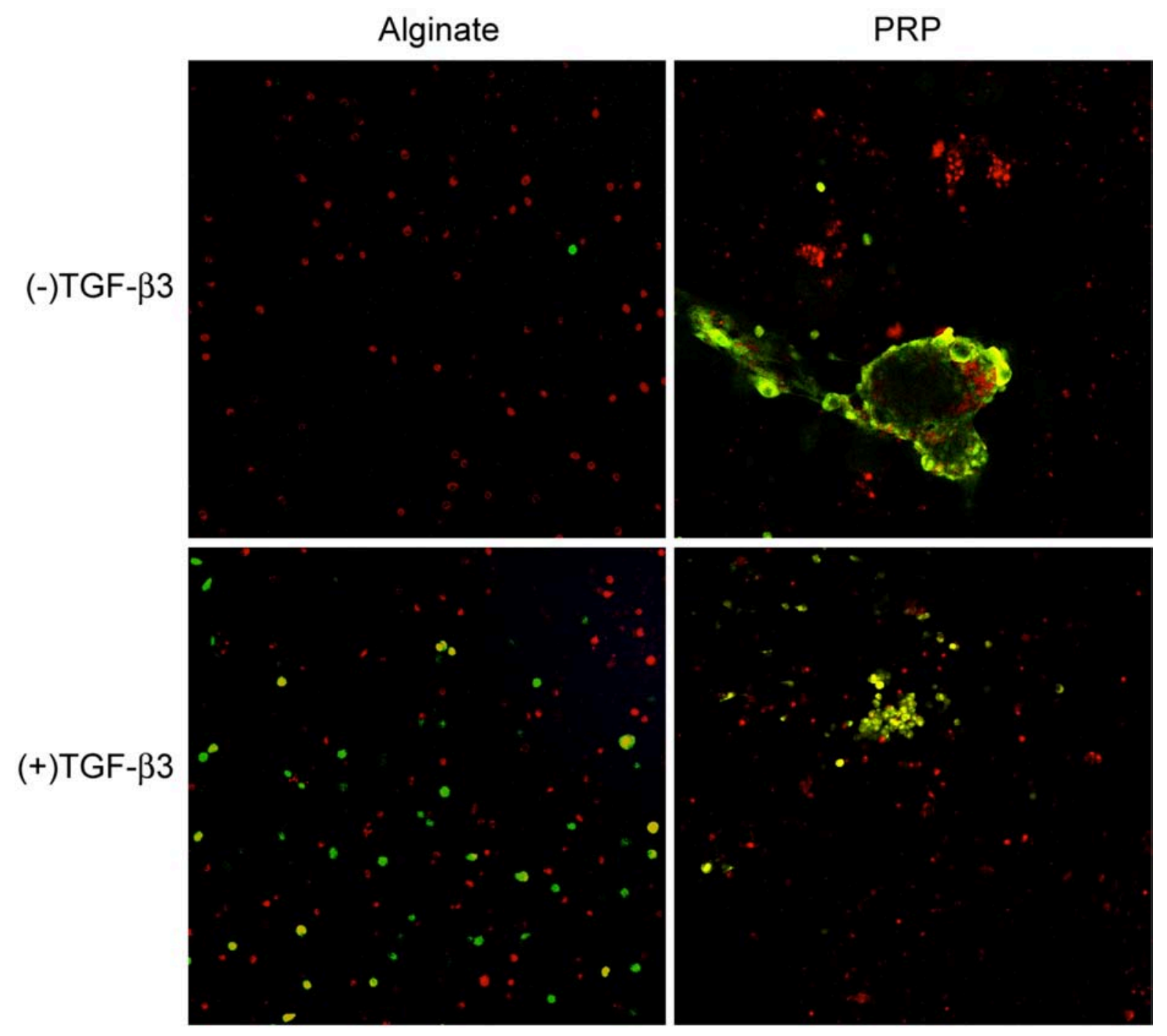

Fig. (1). Fluorescence live-dead imaging of canine MSCs embedded in alginate and PRP and cultured 4 weeks in defined chondrogenic medium. Live cells are red and dead cells are green. 
Sections of the alginate beads were not available for type I collagen staining.

(-)TGF-ß3

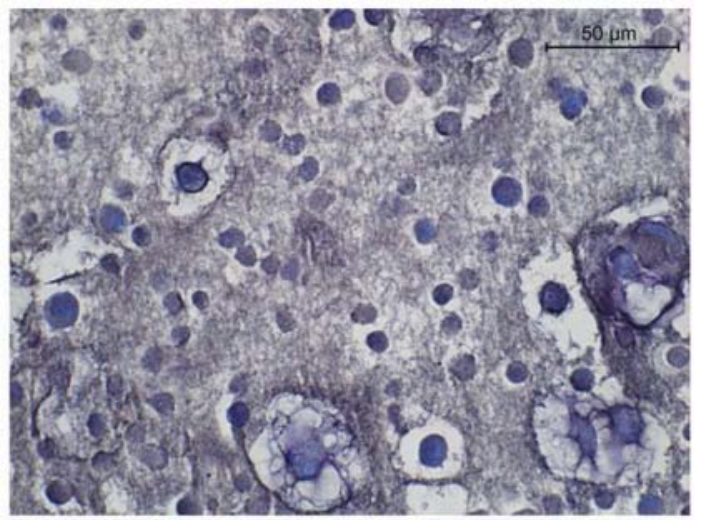

$(+)$ TGF- $\beta 3$

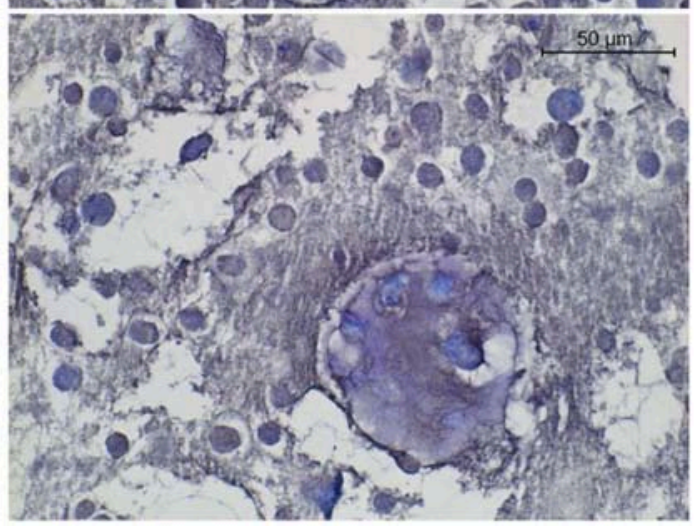

Fig. (2). Toludine blue-stained histological sections of canine MSCs embedded in alginate and cultured 4 weeks in defined chondrogenic medium.
Biochemistry data are summarized in Table 1. In the absence of TGF- $\beta 3$ PRP promoted cell proliferation, but in the presence of $10 \mathrm{ng} / \mathrm{ml}$ TGF- $\beta 3$ PRP beads contained significantly less DNA (fewer cells) than alginate beads. The total amount of GAG produced by cells in alginate was greater than that produced by cells in PRP (data not shown), and TGF- $\beta 3$ significantly increased GAG accumulation by cells in alginate and PRP. However, GAG content normalized to DNA content was not significantly different between PRP and alginate. One clear effect of PRP was on the amount of total collagen normalized to DNA content. It was significantly higher in PRP compared to alginate, both in the absence and presence of TGF- $\beta 3$.

\section{DISCUSSION}

The purpose of this study was to investigate the potential for PRP to stimulate chondroinduction of MSCs in a threedimensional culture model of the type often employed for cartilage tissue engineering. PRP is abundant in mitogenic growth factors such as TGF- $\beta 1$ and PDGF and was therefore expected to increase cell proliferation in our study. In the absence of TGF- $\beta 3$ the stimulatory effect of PRP on cell proliferation in hydrogel culture was similar to the previously reported effect on proliferation in monolayer culture [14-16]. However, PRP did not increase cell number when TGF- $\beta 3$ was present. In fact, the addition of TGF- $\beta 3$ to PRP beads seemed to suppress cell proliferation. This could have occurred because more cells were induced to switch from proliferation to differentiation toward various phenotypic lineages, including but not limited to chondrocytes.

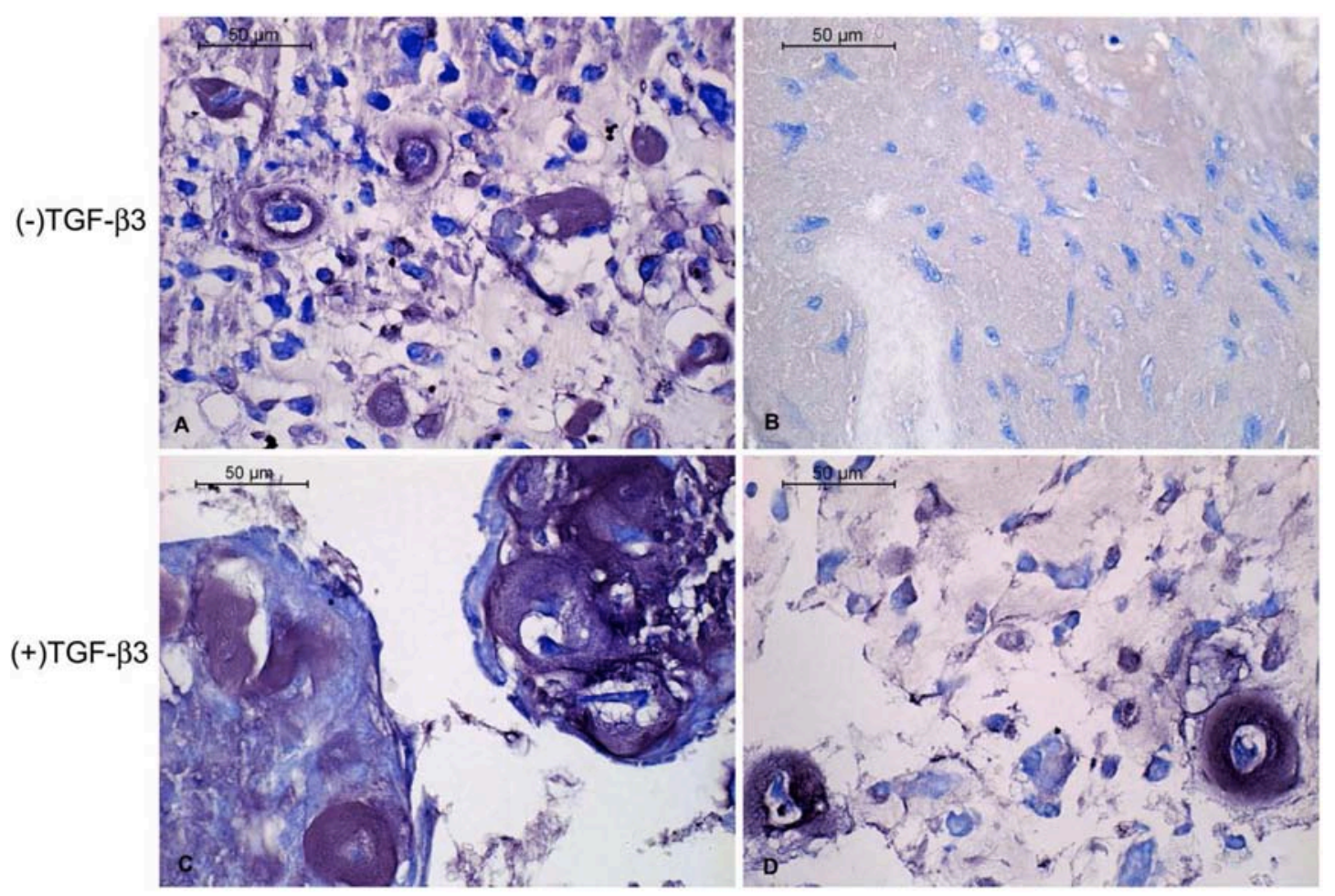

Fig. (3). Toluidine blue-stained histological sections of canine MSCs embedded in PRP/alginate gel and cultured 4 weeks in defined chondrogenic medium. Chondrogenesis was apparent in some regions $(\mathbf{A}, \mathbf{C})$ and much less so in others $(\mathbf{B}, \mathbf{D})$. 


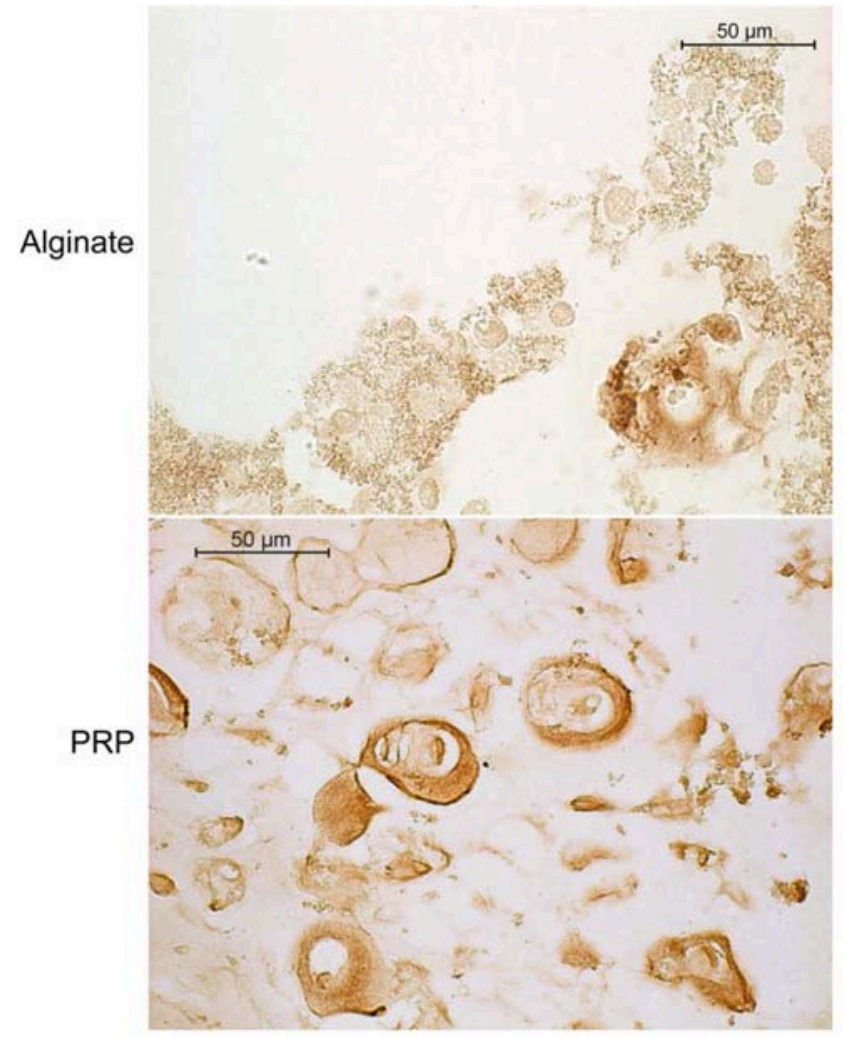

Fig. (4). Type II collagen immunohistochemistry of canine MSCs embedded in alginate or PRP gel and cultured 4 weeks in defined chondrogenic medium containing TGF- $\beta 3$. Positive staining is indicated by a reddish brown color.

We further assessed the ability of PRP to stimulate chondroinduction of primary canine marrow-derived mesenchymal stem cells, alone and in combination with TGF- $\beta 3$. The results did not support our hypothesis. By itself, PRP was a very weak stimulus for chondroinduction and was inferior even to alginate alone. In the presence of chondroinductive TGF- $\beta 3$, PRP and alginate were equivalent in terms of their overall ability to support chondrogenesis. Histology suggested there may have been a qualitative difference, though. The PRP beads contained fewer but larger foci of chondrogenesis, in which clusters of cells were surrounded by more ECM than those found in alginate. Cells in PRP may have gone through a condensation phase in the same way that mesenchymal precursors initiate cartilage development during embryogenesis, and increased cell-cell contact may have enhanced chondrogenesis. It is also possible that growth factors in the PRP were insufficient to induce differentiation but were nonetheless potent enough to stimulate ECM synthesis.

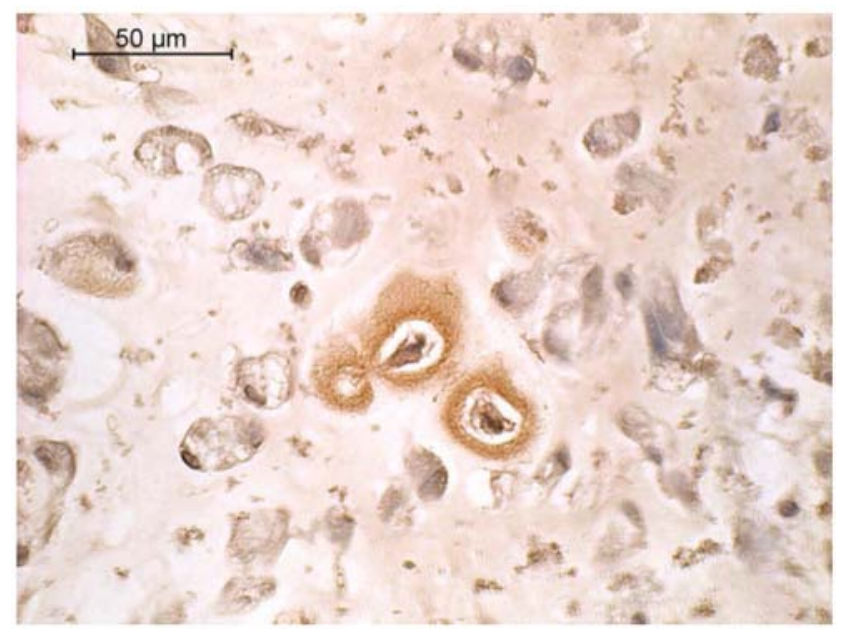

Fig. (5). Type I collagen immunohistochemistry of canine MSCs embedded in PRP/alginate gel and cultured 4 weeks in defined chondrogenic medium without TGF- $\beta 3$. Positive staining is indicated by a reddish brown color.

TGF- $\beta 3$ was not absolutely required for chondroinduction of primary canine bone marrow MSCs, but chondroinduction was greatly enhanced by addition of TGF- $\beta 3$ to MSCs in alginate and PRP. The alginate bead culture system has been demonstrated to promote efficient chondroinduction of MSCs in vitro, and was thus selected to provide a basis for comparison to PRP [28, 29]. Cells do not have receptors for alginate molecules, and they are held in a rounded shape until they synthesize a pericellular matrix with which to establish connections [30]. This spherical morphology encourages chondrogenic differentiation [31]. On the other hand, integrin-mediated cell-ECM interaction as afforded by PRP is an important aspect of TGF- $\beta 3$ induced chondrogenic differentiation [32]. This might explain why the effect of TGF- $\beta 3$ on chondrogenesis was more pronounced in PRP than in alginate.

It is notable that PRP beads contained significantly more collagen than alginate beads. Cartilage ECM is composed mainly of collagen and GAG, and both contribute to the tissue's biomechanics. Tissue engineered cartilage tends to have a lower collagen:GAG ratio than native hyaline cartilage, and increasing the proportion of collagen has been shown to enhance the mechanical properties of engineered cartilage [33, 34]. Thus PRP may be beneficial for raising the collagen content of tissue engineered cartilage. The ECM surrounding cells in PRP beads contained both collagen type I and II. As tissue engineered cartilage matures in vitro, the percentage of collagen type II to total collagen steadily increases [35]. In tissue self-assembled by calf articular

Table 1. DNA, GAG, and collagen content of three-dimensional canine MSC cultures after four weeks of exposure to PRP and TGF- $\beta 3$. Asterisks indicate a statistically significant difference between PRP and alginate $(\mathbf{p}<0.05)$.

\begin{tabular}{|c|c|c|c|c|}
\cline { 2 - 5 } \multicolumn{1}{c|}{} & \multicolumn{2}{c|}{ Alginate } & \multicolumn{2}{c|}{ Alginate } \\
\cline { 2 - 5 } \multicolumn{1}{c|}{} & \multicolumn{2}{|c|}{ Without TGF- $\beta 3$} & \multicolumn{2}{c|}{ With $10 \mathrm{ng} / \mathrm{ml}$ TGF- $\beta 3$} \\
\hline DNA $(\mu \mathrm{g})$ & $1.08 \pm 0.14$ & $1.28 \pm 0.36^{*}$ & $1.20 \pm 0.16$ & $0.88 \pm 0.34^{*}$ \\
\hline GAG $(\mu \mathrm{g} / \mu \mathrm{g}$ DNA $)$ & $9.66 \pm 1.50$ & $7.96 \pm 2.18$ & $14.97 \pm 1.95$ & $13.13 \pm 5.10$ \\
\hline Collagen $(\mu \mathrm{g} / \mu \mathrm{g}$ DNA $)$ & $0.54 \pm 0.14$ & $0.94 \pm 0.33^{*}$ & $0.65 \pm 0.14$ & $1.09 \pm 0.34^{*}$ \\
\hline
\end{tabular}


chondrocytes, the percentage of collagen type II to total collagen was less than $50 \%$ at 4 weeks. So the presence of collagen types I and II is consistent with that of articular cartilage neotissue grown in vitro.

The clinical efficacy of PRP for osteochondral repair remains uncertain. Promising results include the successful resurfacing of an osteochondral rabbit knee defect by synovial membrane-derived MSCs encapsulated in PRP gel and improved articular cartilage repair following intraarticular injection of muscle derived stem cells mixed with PRP in a rat model of chemically induced osteoarthritis [36, 37]. However, some results indicated that PRP has undesirable effects on chondroinduction, such as suppression of collagen type II mRNA expression by MSCs and chondrocytes in vitro [25]. Such disparate results are well documented in a recent review of in vitro and in vivo basic science studies [38]. About $70 \%$ of the studies reviewed concluded that PRP has the potential to upregulate chondrogenic gene expression as well as increase the production of proteoglycan and type II collagen, while the other studies found the effects of PRP to be negligible or disadvantageous. The total body of evidence was considered to comprise a proof of concept for the clinical use of PRP. Our data indicate that efficient chondroinduction of MSCs is not achieved with PRP only moderately enriched in platelets. The platelet concentration was only about 2.5 times that of whole blood, which may have insufficiently concentrated the growth factors platelets harbor. Increasing the platelet concentration to approximately 5 -fold that of whole blood is anticipated to amplify PRP's effects. Another limitation of the current study was that our PRP was not adjusted to neutral $\mathrm{pH}$, and it did appear to decrease the $\mathrm{pH}$ of the culture medium based on observed changes in color of the phenol-red containing culture medium. PRP buffered to neutral $\mathrm{pH}$ using sodium bicarbonate has been demonstrated to enhance MSC proliferation and chondrogenic differentiation [23].

\section{CONCLUSION}

Although the current study did not demonstrate a strong chondroinductive effect of PRP on marrow-derived MSCs, the results suggest that it may stimulate collagen production and enhance the chondrogenic effect of exogenous TGF- $\beta 3$.

\section{CONFLICT OF INTEREST}

The authors confirm that this article content has no conflict of interest.

\section{ACKNOWLEDGEMENTS}

The II-II6B3 monoclonal antibody developed by T.F. Linsenmayer was obtained from the Developmental Studies Hybridoma Bank developed under the auspices of the NICHD and maintained by The University of Iowa, Department of Biology, Iowa City, IA 52242.

\section{REFERENCES}

[1] Frojmovic M, Milton J. Human platelet size, shape, and related functions in health and diseases. Physiol Rev 1982; 62(1): 185-261.
[2] Lu H, Vo J, Chin H, et al. Controlled delivery of platelet-rich plasma-derived growth factors for bone formation. J Biomed Mater Res A 2008; 86(4): 1128-36.

[3] Wei L, Lei G, Sheng P, et al. Efficacy of platelet-rich plasma combined with allograft bone in the management of displaced intra-articular calcaneal fractures: A prospective cohort study. J Orthop Res 2012; 30(10): 1570-6.

[4] Thor A, Hong J, Kjeller G, Sennerby L, Rasmusson L. Correlation of platelet growth factor release in jawbone defect repair - A study in the dog mandible. Clin Implant Dent Relat Res 2013; 15(5): 75968.

[5] Owens R, Ginetti J, Conti S, Latona C. Clinical and magnetic resonance imaging outcomes following platelet rich plasma injection for chronic midsubstance Achilles tendinopathy. Foot Ankle Int 2011; 32(11): 1032-9.

[6] de Almeida A, Demange M, Sobrado M, Rodrigues M, Pedrinelli A, Hernandez A. Patellar tendon healing with platelet-rich plasma: a prospective randomized controlled trial. Am J Sports Med 2012; 40(6): 1282-8.

[7] Murray M, Palmer M, Abreu ESK, Zurakowski D, Fleming B. Platelet-rich plasma alone is not sufficient to enhance suture repair of the ACL in skeletally immature animals: an in vivo study. J Orthop Res 2009; 27(5): 639-45.

[8] Waselau M, Sutter W, Genovese R, Bertone A. Intralesion injection of platelet-rich plasma followed by controlled exercise for treatment of midbody suspensory ligament desmitis in Standardbred racehorses. J Am Vet Med Assoc 2008; 232(10): 151-20.

[9] Hammond J, Hinton R, Curl L, Muriel J, Lovering R. Use of autologous platelet-rich plasma to treat muscle strain injuries. Am J Sports Med 2009; 37(6): 1135-42.

[10] Milano G, SannaPassino E, Deriu L, et al. The effect of platelet rich plasma combined with microfractures on the treatment of chondral defects: an experimental study in a sheep model. Osteoarthritis Cartilage 2010; 18(7): 971-80.

[11] Qi Y, Chen X, Jiang Y, et al. Local delivery of autologous platelet in collagen matrix simulated in situ articular cartilage repair. Cell Transplant 2009; 18(10): 1161-9.

[12] Kon E, Filardo G, Delcogliano M, et al. Platelet autologous growth factors decrease the osteochondral regeneration capability of a collagen-hydroxyapatite scaffold in a sheep model. BMC Musculoskelet Disord 2010; 11: 220.

[13] Wu W, Chen F, Liu Y, Ma Q, Mao T. Autologous injectable tissueengineered cartilage by using platelet-rich plasma: experimental study in a rabbit model. J Oral Maxillofac Surg 2007; 65(10): 1951-7.

[14] Lucarelli E, Beccheroni A, Donati D, et al. Platelet-derived growth factors enhance proliferation of human stromal stem cells. Biomaterials 2003; 24(18): 3095-100.

[15] Chieregato K, Castegnaro S, Madeo D, Astori G, Pegoraro M, Rodeghiero F. Epidermal growth factor, basic fibroblast growth factor and platelet-derived growth factor-bb can substitute for fetal bovine serum and compete with human platelet-rich plasma in the ex vivo expansion of mesenchymal stromal cells derived from adipose tissue. Cytotherapy 2011; 13(8): 933-43.

[16] Goedecke A, Wobus M, Krech M, et al. Differential effect of platelet-rich plasma and fetal calf serum on bone marrow-derived human mesenchymal stromal cells expanded in vitro. J Tissue Eng Regen Med 2011; 5(8): 648-54.

[17] Vogel J, Szalay K, Geiger F, Kramer M, Richter W, Kasten P. Platelet-rich plasma improves expansion of human mesenchymal stem cells and retains differentiation capacity and in vivo bone formation in calcium phosphate ceramics. Platelets 2006; 17(7): 462-9.

[18] Parsons P, Butcher A, Hesselden K, et al. Platelet-rich concentrate supports human mesenchymal stem cell proliferation, bone morphogenetic protein-2 messenger RNA expression, alkaline phosphatas activity, and bone formation in vitro: a mode of action to enhance bone repair. J Orthop Trauma 2008; 22(9): 595-604.

[19] Lin S, Landesberg R, Chin H, Lin J, Eisig S, Lu H. Controlled release of PRP-derived growth factors promotes osteogenic differentiation of human mesenchymal stem cells. Conf Proc IEEE Eng Med Biol Soc 2006; 1: 4358-61.

[20] Shin S, Yoo J, Kim H, Nam J, Kim H. Enhanced cellular responses of human bone marrow stromal cells cultured on pre-treated 
surface with allogenic platelet-rich plasma. Connect Tissue Res 2012; 53(4): 318-26.

[21] Arpornmaeklong P, Kochel M, Depprich R, Kubler N, Wurzler K. Influence of platelet-rich plasma (PRP) on osteogenic differentiation of rat bone marrow stromal cells. Int $\mathrm{J}$ Oral Maxillofac Surg 2004; 33(1): 60-70.

[22] Mooren R, Hendricks E, van den Beucken J, et al. The effect of platelet-rich plasma in vitro on primary cells: rat osteoblast-like cells and human endothelial cells. Tissue Eng Part A 2010; 16(10): 3159-72.

[23] Mishra A, Tummala P, King A, et al. Buffered platelet-rich plasma enhances mesenchymal stem cell proliferation and chondrogenic differentiation. Tissue Eng Part C Methods 2009; 15(3): 431-5.

[24] Kruger J, Hondke S, Endres M, Pruss A, Siclari A, Kaps C. Human platelet-rich plasma stimulates migration and chondrogenic differentiation of human subchondral progenitor cells. J Orthop Res 2012; 30(6): 845-52.

[25] Drengk A, Zapf A, Sturmer E, Sturmer K, Frosch K. Influence of platelet-rich plasma on chondrogenic differentiation and proliferation of chondrocytes and mesenchymal stem cells. Cells Tissues Organs 2009; 189(5): 317-26.

[26] Hoemann C. Molecular and biochemical assays of cartilage components. Methods Mol Med 2004; 101: 127-56.

[27] Reddy G, Enwemeka C. A simplified method for the analysis of hydroxyproline in biological tissues. Clin Biochem 1996; 29(3): 225-9.

[28] Yang I, Kim S, Kim Y, Sun H, Kim S, Lee J. Comparison of phenotypic characterization between "alginate bead" and "pellet" culture systems as chondrogenic differentiation models for human mesenchymal stem cells. Yonsei Med J 2004; 45(5): 891-900.

[29] Ma H, Hung S, Lin S, Chen Y, Lo W. Chondrogenesis of human mesenchymal stem cells encapsulated in alginate beads. J Biomed Mater Res A 2003; 64(2): 273-81.
[30] Lee K, Kong H, Mooney D. Quantifying interactions between cell receptors and adhesion ligand-modified polymers in solution. Macromol Biosci 2008; 8(2): 14-5.

[31] Mathieu P, Loboa E. Cytoskeletal and focal adhesion influences on mesenchymal stem cells shape, mechanical properties, and differentiation down osteogenic, adipogenic, and chondrogenic pathways. Tissue Eng Part B Rev 2012; 18(6): 436-44.

[32] Chang JC, Hsu Sh, Chen D. The promotion of chondrogenesis in adipose-derived adult stem cells by an RGD-chimeric protein in 3D alginate culture. Biomaterials 2009; 30(31): 6265-75.

[33] Williamson A, Masuda K, Thonar E, Sah R. Growth of immature articular cartilage in vitro: correlated variation in tensile biomechanical and collagen network properties. Tissue Eng 2003; 9: 625-34.

[34] Asanbaeva A, Masuda K, Thonar E, Klisch S, Sah R. Mechanisms of cartilage growth: modulation of balance between proteoglycan and collagen in vitro using chondroitinase ABC. Arthritis Rheum 2007; 56: 188-98.

[35] Ofek G, Revell C, Hu J, Allison D, Grande-Allen K, Athanasiou K. Matrix development in self-assembly of articular cartilage. PLoS One 2008; 3(7): e2795.

[36] Lee J, Min H, Park H, Lee S, Seong S, Lee M. Synovial membrane-derived mesenchymal stem cells supported by plateletrich plasma can repair osteochondral defects in a rabbit model. Arthroscopy 2013; 29(6): 1034-46.

[37] Mifune Y, Matsumoto T, Takayama K, et al. The effect of plateletrich plasma on the regenerative therapy of muscle derived stem cells for articular cartilage repair. Osteoarthritis Cartilage 2013; 21(1): $175-85$.

[38] Smyth N, Murawski C, Fortier L, Cole B, Kennedy J. Platelet-rich plasma in the pathologic processes of cartilage: review of basic science evidence. Arthroscopy 2013; 29(8): 1399-409.

(C) Elder and Thomason; Licensee Bentham Open.

This is an open access article licensed under the terms of the Creative Commons Attribution Non-Commercial License (http://creativecommons.org/licenses/by-nc/3.0/) which permits unrestricted, non-commercial use, distribution and reproduction in any medium, provided the work is properly cited. 